\title{
Perfil sociodemográfico e uso de produtos naturais em pacientes com a síndrome do pé diabético na ala vascular de um hospital público de Alagoas, Brasil
}

\author{
Sociodemographic profile and use of natural products in patients with diabetic foot syndrome in
} the vascular wing of a public hospital in Alagoas, Brazil

Perfil sociodemográfico y uso de productos naturales en pacientes con síndrome de pie diabético en el ala vascular de un hospital público de Alagoas, Brasil

\section{Resumo}

O diabetes é uma desordem metabólica com alta prevalência em toda população mundial e elevada morbidade, decorrente das suas complicações nos sistemas cardiovascular e neurológico. Entre as complicações mais evidentes no cotidiano clínico, encontra-se as feridas, insidiosas e de difícil cicatrização, que evoluem para a amputação, inicialmente de um pododáctilo, podendo até progredir para a desarticulação coxofemoral. É conhecido pela sabedoria popular as propriedades de determinados produtos naturais para o tratamento de feridas, em diferentes apresentações, 
por ação dos seus compostos bioativos. O objetivo deste estudo foi analisar o perfil dos pacientes e conhecer produtos naturais que são utilizados no tratamento da síndrome do pé diabético. Trata-se de um estudo de coorte transversal, por meio de uma entrevista aos pacientes portadores de úlcera do pé diabético, com perguntas sobre o aspecto social e demográfico, tratamento e evolução sintomatológica. Para análise estatística foram utilizados os programas Excel® e BioEstat 5.0®. Foi observada uma mudança no perfil destes pacientes comparadas a estudos anteriores, mostrando um aumento da prevalência em uma população com maior nível de escolaridade e inferiu a prevalência no sexo feminino, corroborando com outros estudos. Além disso, constatou-se que a maior parte havia sido diagnosticada há pelo menos 10 anos e que a maioria é composta por trabalhadores do serviço e comércio. Compreende-se a necessidade da criação de equipes multidisciplinares para o controle da diabetes e a identificação precoce do pé diabético, além da necessidade de capacitação das equipes na atenção especializada.

Palavras-chave: Angiopatias diabéticas; Perfis sanitários; Produtos biológicos; Epidemiologia.

\begin{abstract}
Diabetes is a metabolic disorder with a high prevalence throughout the world population and high morbidity due to its complications in the cardiovascular and neurological systems. Among the complications most evident in the clinical routine are the wounds, insidious and difficult to heal, that evolves to the amputation, initially of a polydactyl, and may even progress to the hip joint disarticulation. It is widespread among the world that the properties of certain natural products for the treatment of wounds, in different presentations, by the action of its bioactive compounds. The aim of this study was to analyze the profile of patients and to know the natural products that are being used in the treatment of diabetic foot syndrome. This is a cross-sectional cohort study, through an interview with patients with diabetic foot ulcers, with questions about the social and demographic aspects, treatment and symptomatic evolution. For statistical analysis, the Excel ${ }^{\circledR}$ and BioEstat $5.0 \AA$ programs were used. It was observed a change in the profile of these patients compared to previous studies, showing an increase in prevalence in a population with higher level of education and also was observed the prevalence in the female sex, corroborating with other studies. In addition, it was found that most of them had been diagnosed at least 10 years ago and that most of them are service and commerce workers. It is understood the need to create a multidisciplinary healthcare team to control diabetes and early identification of diabetic foot, as well as the need for training of teams in specialized care.
\end{abstract}

Keywords: Diabetic angiopathies; Sanitary profiles; Biological products; Epidemiology.

\title{
Resumen
}

La diabetes es un trastorno metabólico con alta prevalencia en toda la población mundial y alta morbilidad, resultante de sus complicaciones en los sistemas cardiovascular y neurológico. Entre las complicaciones más evidentes en la práctica clínica diaria se encuentran las heridas, insidiosas y de difícil cicatrización, que progresan a la amputación, inicialmente de un dedo del pie, pudiendo incluso progresar a la desarticulación de la cadera. Es conocida por la sabiduría popular las propiedades de ciertos productos naturales para el tratamiento de heridas, en diferentes presentaciones, por la acción de sus compuestos bioactivos. El objetivo de este estudio fue analizar el perfil de los pacientes y conocer los productos naturales que se utilizan en el tratamiento del síndrome del pie diabético. Se trata de un estudio de cohorte transversal, mediante entrevista a pacientes con úlceras del pie diabético, con preguntas sobre los aspectos sociodemográficos, el tratamiento y la evolución sintomática. Para el análisis estadístico se utilizaron los programas Excel® y BioEstat 5.0®. Se observó un cambio en el perfil de estos pacientes con respecto a estudios previos, mostrando un aumento de la prevalencia en una población con mayor nivel educativo y se infirió la prevalencia en el sexo femenino, corroborando con otros estudios. Además, se constató que la mayoría de ellos habían sido diagnosticados hace al menos 10 años y que la mayoría son trabajadores de servicios y comercio. Se entiende la necesidad de crear equipos multidisciplinares para el control de la diabetes y la identificación precoz del pie diabético, además de la necesidad de formación de equipos en atención especializada.

Palabras clave: Angiopatías diabéticas; Perfiles sanitarios; Productos biológicos; Epidemiología.

\section{Introdução}

O Diabetes é um grupo de distúrbios metabólicos complexos caracterizados por deficiência na secreção e redução da ação da insulina ou uma combinação de ambos resultando em hiperglicemia (Crawford et al., 2017). O aumento das taxas de sobrepeso e obesidade associado às alterações do estilo de vida e ao envelhecimento populacional, são os principais fatores que explicam o crescimento da prevalência do diabetes (Sartorelli et al., 2003; Franco et al., 2008). Além disso, um dos maiores e mais graves problemas de indivíduos diabéticos é o desenvolvimento de úlceras na extremidade inferior, geralmente precursoras da amputação, que iniciam pela neuropatia e subsequentes surgimento de feridas no pé, sendo um problema de saúde pública cada vez mais relevante (Caiafa et al., 2011). 
O uso de plantas e produtos de origem natural pode ser entendido como uma prática que atravessa milênios, estando historicamente presente na sabedoria do senso comum, articulando cultura e saúde, uma vez que estes aspectos não ocorrem isoladamente, mas inseridos em um contexto histórico determinado (Alvim et al., 2006). Sagbo et al. (2017) descreveram que as plantas do gênero Brachylaena sob forma de extrato com álcool apresentam atividade antioxidante, contendo uma grande variedade de moléculas que colaboram na eliminação de radicais livres tais como fenóis, taninos, alcaloides e saponinas.

Diante do exposto, conhecer o perfil epidemiológico desses pacientes contribuirá na elaboração de medidas públicas, como nos programas e estratégias da atenção básica de saúde e na elaboração de novos fármacos e produtos biotecnológicos que possam tratar as feridas e úlceras do pé diabético.

\section{Metodologia}

Caracterizou-se como um estudo qualitativo do tipo coorte transversal. Segundo Pereira et al. (2018), os métodos qualitativos são aqueles nos quais é importante a interpretação por parte do pesquisador sobre o fenômeno em estudo, utilizando-se entrevistas com questões abertas para a coleta de dados.

O presente estudo foi desenvolvido com a concessão e aprovação do comitê de ética da Universidade Estadual de Ciências da Saúde de Alagoas - UNCISAL registrado sob o parecer consubstanciado 2.118.489 e aprovação com termo de anuência do Hospital Geral do Estado - Professor Osvaldo Brandão Vilela (HGE). Foi realizada uma entrevista com os pacientes que se adequaram aos critérios de inclusão e que aceitaram participar do estudo com respectivo aval por meio da assinatura do termo de consentimento livre e esclarecido. Foram os seguintes critérios de inclusão: indiferente ao gênero; ter idade igual ou superior a 18 anos, consciente e lúcido, possuir diabetes tipo 2, apresentar úlcera do pé diabético.

A coleta de dados foi realizada durante seis meses com pacientes na ala vascular do HGE. Cada paciente foi identificado por meio de um código numérico, sendo o cabeçalho de identificação preenchido com as informações referentes à idade, sexo, procedência e profissão atual. O questionário da entrevista continha 14 perguntas sobre os aspectos sociais e demográficos, fisiopatologia e tratamento, guiadas pelos seguintes itens: há quanto tempo havia o diagnóstico de diabetes, se há alguma outra doença crônica associada, se é adepto às práticas de exercício físico e sua frequência, grau de escolaridade, o ramo de atuação profissional, se há outros casos de diabetes entre os genitores, qual o sintoma mais relevante que o levou a buscar ajuda médica para o diagnóstico do diabetes, se conhece algum tipo de produto natural e se já empregou para o tratamento de feridas com aplicação na síndrome do pé diabético, qual a forma de uso de acordo com o conhecimento popular, conhece outro produto natural, que não tenha sido empregado por ele, com ação cicatrizante e que possa ser usado no tratamento das feridas da síndrome do pé diabético, acredita no potencial terapêutico desses produtos naturais e que tipo de perigo acredita que esses produtos possam oferecer.

Para o armazenamento dos dados foi utilizada a planilha Excel 2016 com armazenamento em nuvem e para a construção dos gráficos foi utilizado o programa BioEstat versão 5.0.

\section{Resultados}

Neste estudo a amostra foi determinada por convenção, sendo composta por trinta e quatro pacientes ( $\mathrm{n}=34)$, sendo 17 mulheres e 17 homens selecionados arbitrariamente na ala vascular do Hospital Geral do Estado e que se enquadraram nos critérios de inclusão. A faixa etária predominante compreendeu pacientes na faixa da sétima década de vida, sendo a idade máxima e mínima dos pacientes com 76 e 32 anos, respectivamente. A maior parte do público deste estudo correspondendo a $55,88 \%(n=19)$ tinha o diagnóstico de diabetes há pelo menos 10 anos ou mais, seguido por 20,59\% ( $\mathrm{n}=7$ ) com quadro entre 5 e 10 anos de diagnóstico, além de 17,65\% (n=6) entre 2 e 5 anos. Paciente com diagnóstico entre 1 e 2 anos não foram 
registrados. Apenas dois pacientes apresentaram diagnóstico no último ano, corroborando com 5,88\% dos casos deste quesito, como pode ser visto na Figura 1.

Figura 1. Tempo de diagnóstico do diabetes em anos.

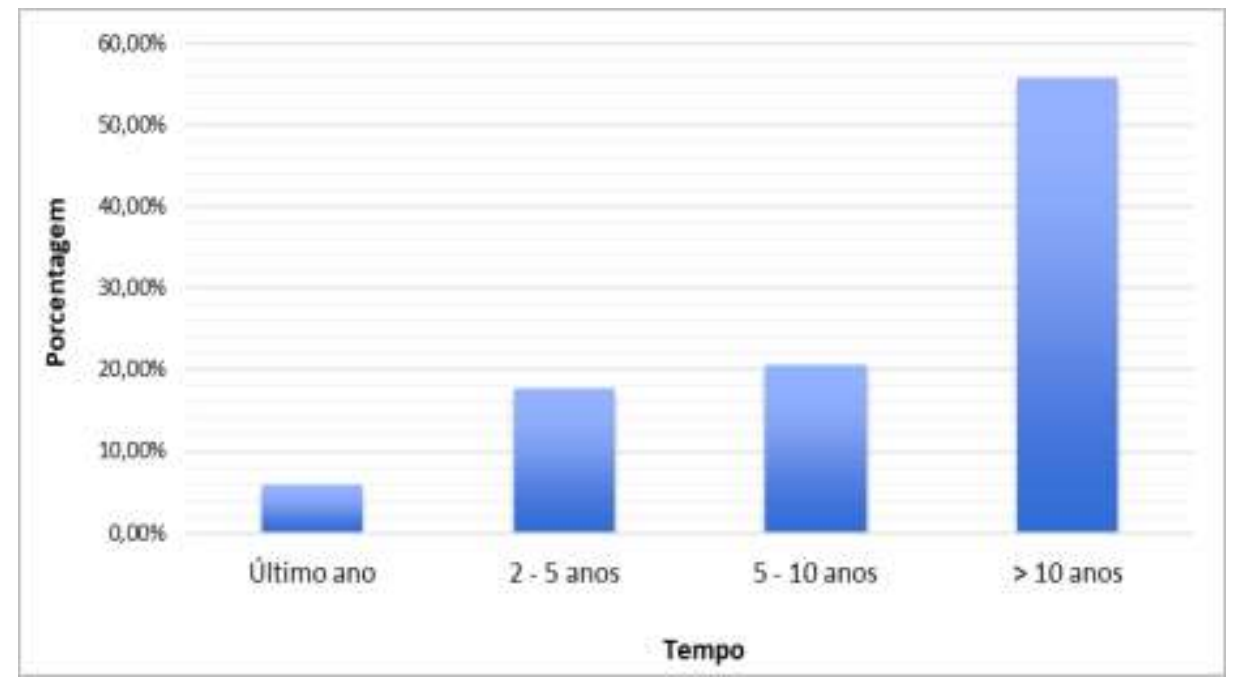

Fonte: Autores.

O segundo quesito do questionário identifica a adesão e frequência da prática de exercícios físicos por parte dos pacientes. 79,41\% ( $\mathrm{n}=27)$ consideraram-se sedentários e não eram adeptos a atividades físicas, os que se exercitavam ao menos uma vez na semana equivalente a $11,76 \%(\mathrm{n}=4)$ e diariamente $8,82 \%(\mathrm{n}=3)$ adeptos a práticas quinzenais não foram identificados, conforme Figura 2.

Figura 2. Adesão à prática de exercícios físicos.

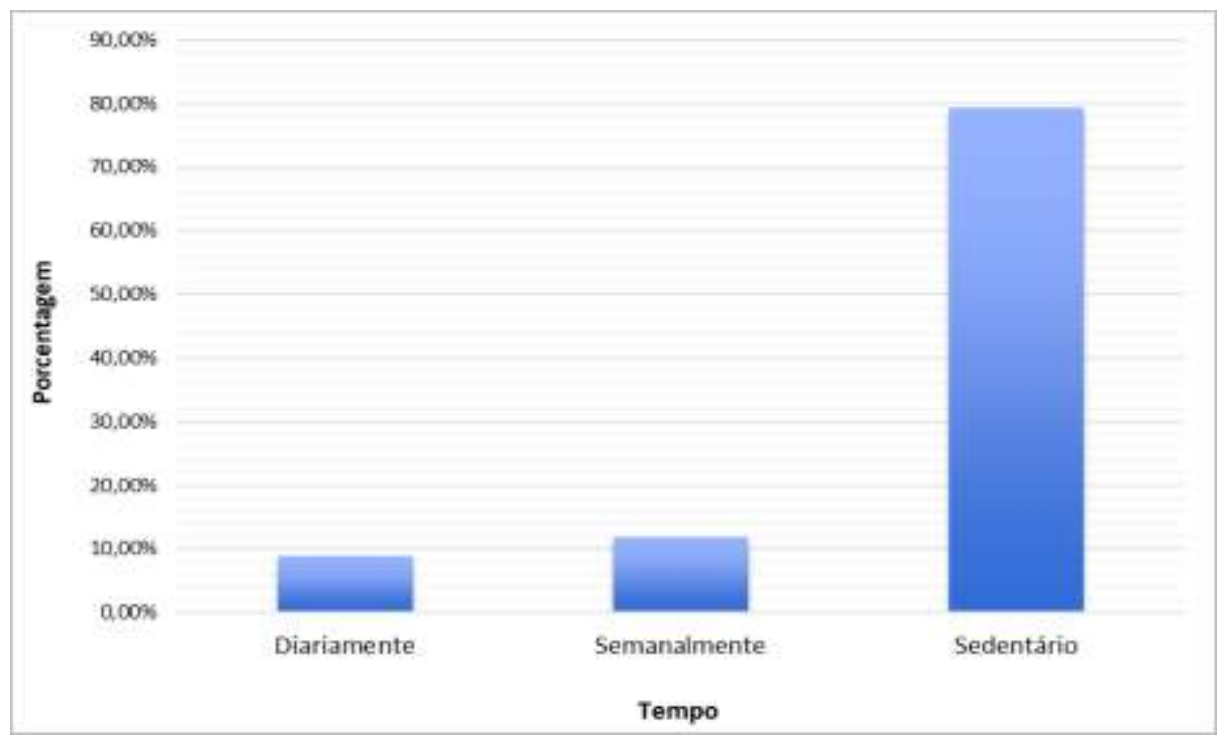

Fonte: Autores.

Do total de pacientes coletados, cada paciente pode apresentar mais de uma patologia associada obtendo o resultado de que 77,14\% ( $\mathrm{n}=27$ ) possuem como doença crônica associada hipertensão arterial sistêmica, seguido de 5,71\% ( $\mathrm{n}=2$ ) que eram nefropatas, $2,86 \%$ que corresponde a apenas um paciente cardiopata e outro com glaucoma que corrobora com $2,86 \%$. 
Do total, apenas cerca de $11,43 \%$ ( $\mathrm{n}=4$ ) dos pacientes disseram não possuir nenhuma doença de natureza crônica associada ao quadro de diabetes (Figura 3).

Figura 3. Doenças crônicas associadas.

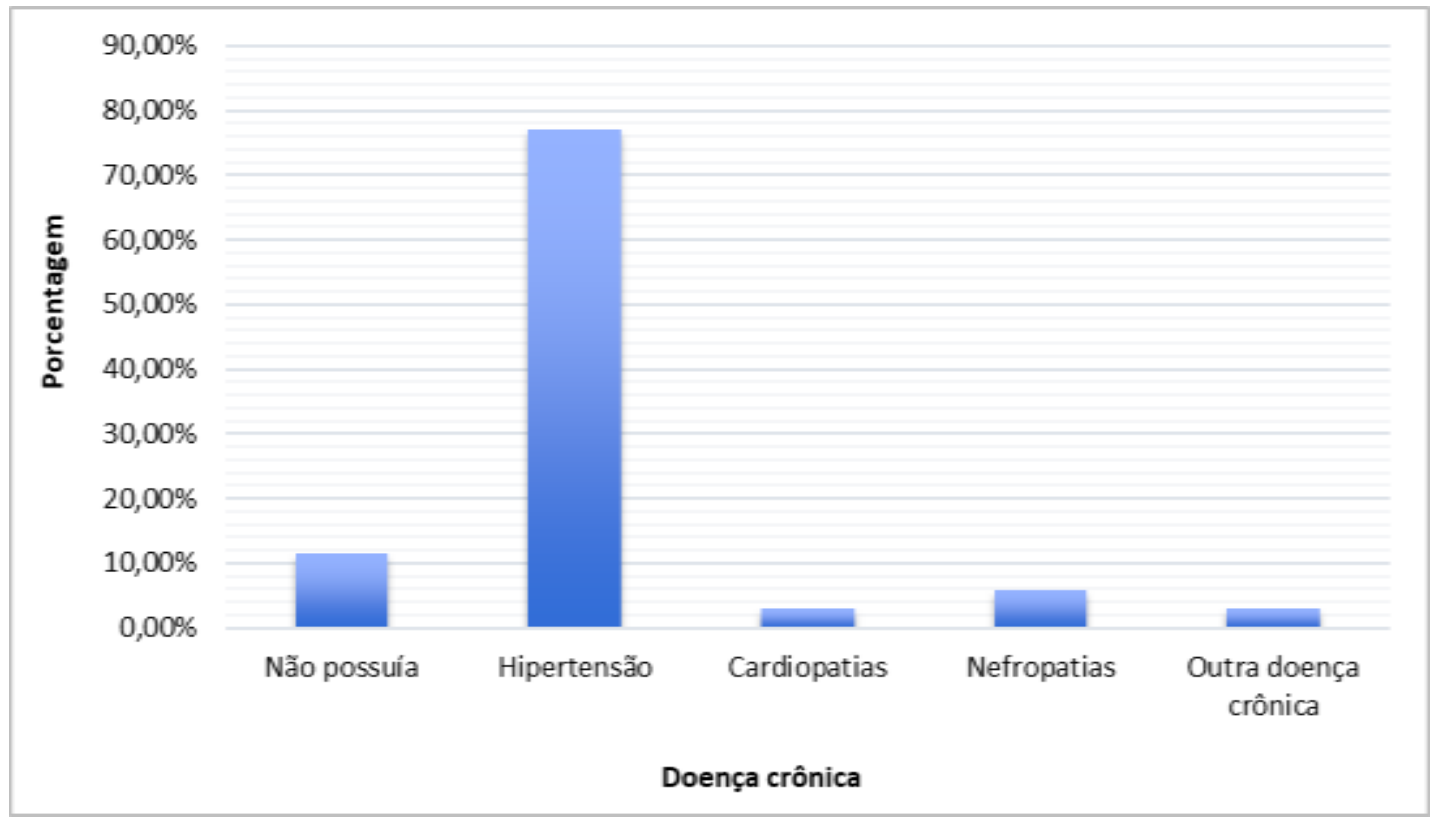

Fonte: Autores.

Sobre o grau de escolaridade do público desse estudo, foi verificado que $29,41 \%$ ( $\mathrm{n}=10$ ) havia concluído o ensino médio, seguido 20,59\% ( $n=7$ ) que possuem o fundamental completo. 20,59\% nunca havia frequentado a escola. Pacientes que não concluíram o fundamental correspondem a $8,82 \%$ (n=3). Os que possuíam médio-técnico e superior incompleto equivalem, isoladamente, a 2,94\% ( $n=1$ ). Neste estudo não foi encontrado pacientes com nível superior completo (Figura 4).

Figura 4. Percentual do nível de escolaridade.

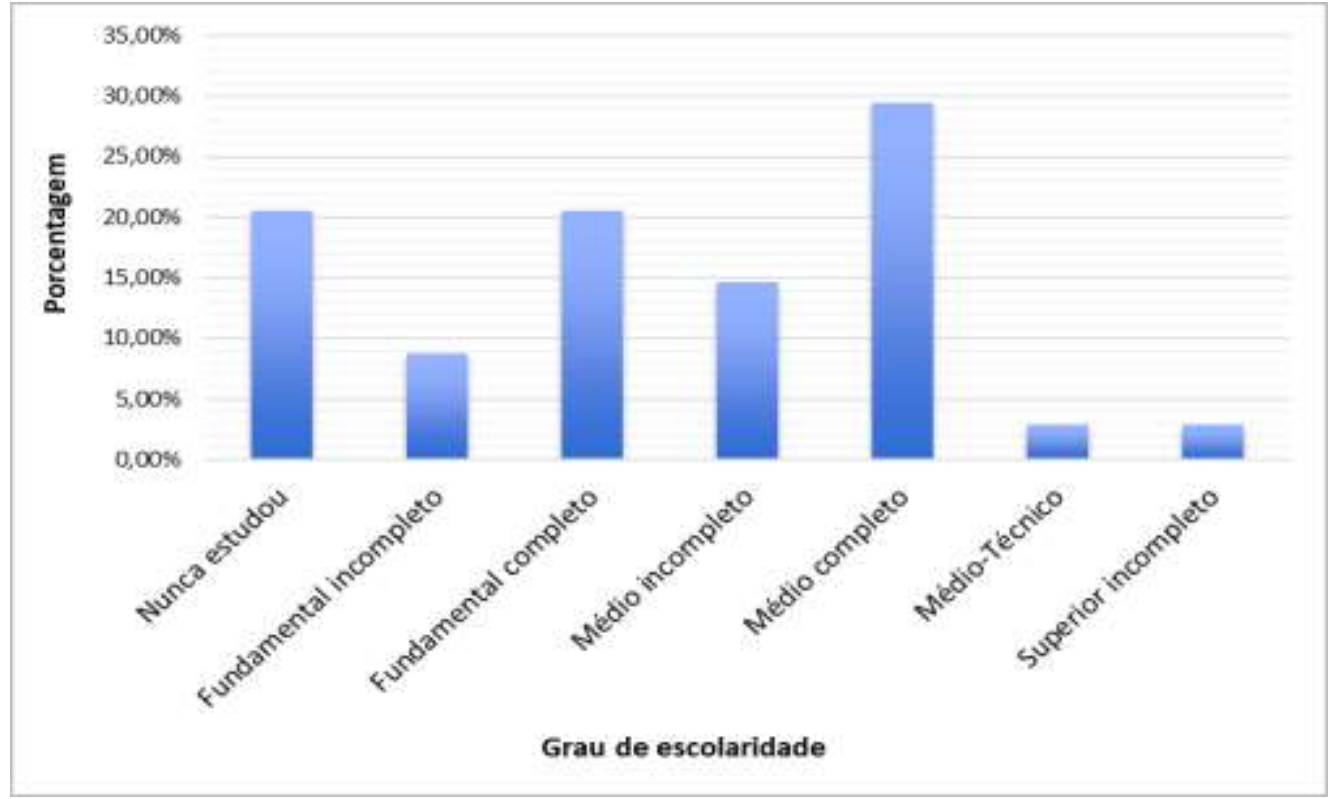

Fonte: Autores. 
O quinto item identifica a atuação profissional do paciente, demonstrando que a grande parte é formada por trabalhadores autônomos com uma porcentagem de 32,35\% ( $\mathrm{n}=11)$, seguido por trabalhadores do setor terciário, que inclui serviço e comércio, que compõe $29,41 \%$ ( $\mathrm{n}=10$ ), logo depois, trabalhadores do campo, enquadrado com 17,65\% ( $\mathrm{n}=6$ ), em seguida com um montante de $14,71 \%(\mathrm{n}=5)$ encaixam-se os servidores públicos e com menor índice neste estudo os trabalhadores da indústria 5,88\% ( $=2)$, de acordo com a Figura 5.

Figura 5. Estratificação do ramo ou setor laboral dos pacientes.

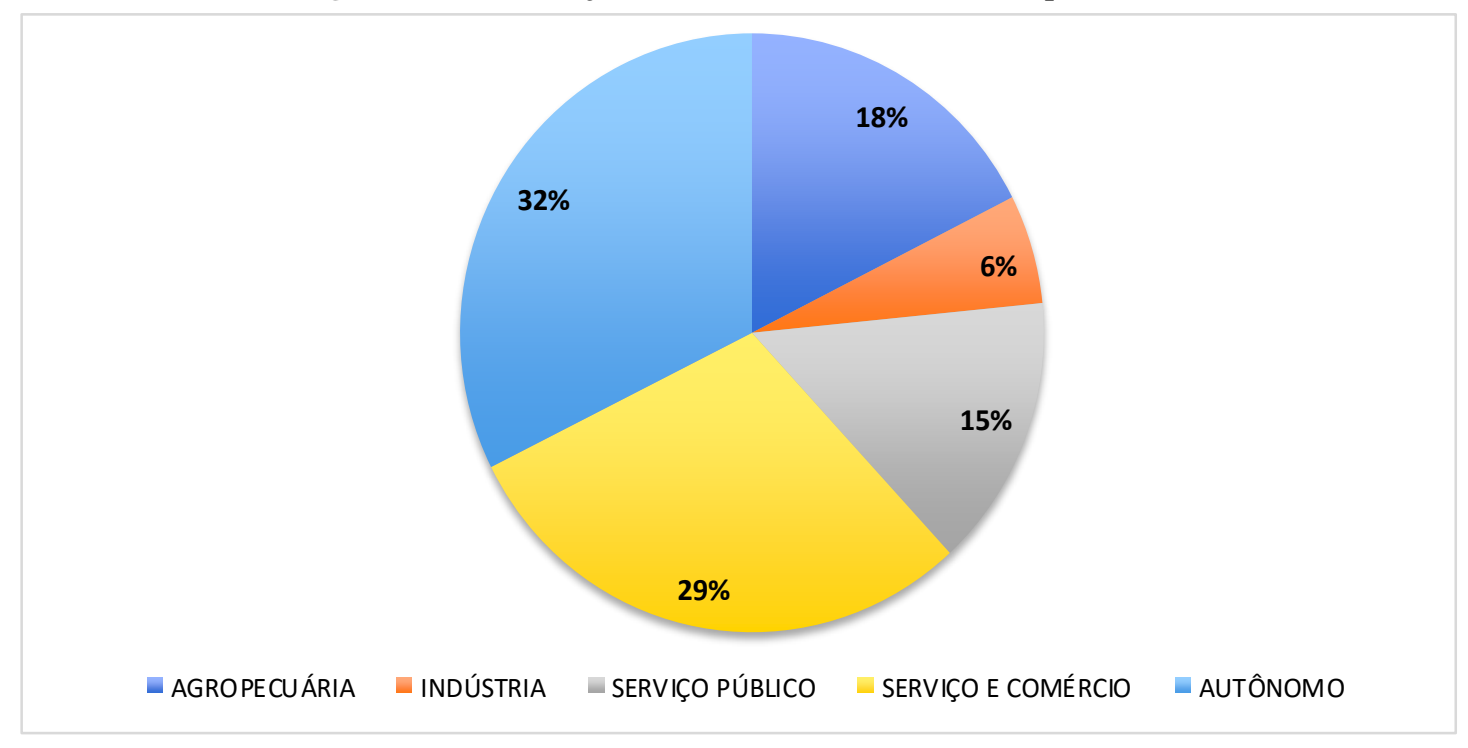

Fonte: Autores.

Em relação ao caso de diabetes entre os pais as alternativas que apresentava as opções de ambos os genitores apresentarem diabetes (entre pai e mãe) correspondeu a $35,29 \%$ dos casos ( $n=12)$, seguido de $26,47 \%$ de caso conhecido apenas na herança paterna $(n=9)$, Os casos que acometeram apenas a mãe é referente a $14,71 \%(n=5)$. Os pacientes que não souberam informar se havia relação de diabete entre os pais também registrou $8,82 \%(n=3) .14,71 \%$ (n=5) não havia casos registrados entre os pais.

O sétimo item identifica qual a manifestação clínica que o paciente recorda ter sido fundamental para o diagnóstico médico do diabetes. 44,12\% ( $\mathrm{n}=15$ ) referiram que o aumento no volume urinário (poliúria) foi a manifestação clínica mais relevante. O segundo sintoma mais evidente foi o ressecamento dos lábios (xerostomia) sendo relatada por 11,76\% ( $\mathrm{n}=4$ ) e o aumento excessivo da sede (polidipsia) registrado em apenas 5,88\% (n=2). Não se recorda do quadro inicial para o diagnóstico correspondeu a $23,53 \%(n=8)$ e $14,71 \%(n=5)$ referiram não ter havido sinais ou sintomas do quadro diabetogênico.

No item sobre o conhecimento e adesão de produto(s) natural(is) a terapêutica de feridas, $29,41 \%$ ( $\mathrm{n}=10$ ) conhecia algum produto com a finalidade e já havia usado ou usaria com a finalidade de tratá-las. $8,82 \%(n=3)$ relataram que conhece algum produto natural, mas que não usou ou que não usaria com tal finalidade. Os pacientes que desconhecem, porém usaria caso tivesse acesso correspondeu 23,53\% dos casos $(\mathrm{n}=8)$ e 11,76\% $(\mathrm{n}=4)$ desconhece e não seria adepto ao uso.

Os pacientes quando questionados a respeito do conhecimento e adesão de produto(s) natural(is) para o tratamento dos sintomas da diabetes - relacionada a melhora do controle glicêmico - as respostas aos itens foram mais diversificadas. Os que referiram que conheciam e usaram ou usariam determinado produto natural com finalidade terapêutica para o controle glicêmico ficou em 20,59\% (n=7). Os que conhecem e não usaram ou não fariam usufruto foi de $8,82 \%$ (n=3) sendo essa parcela do estudo a minoria percentual e absoluta. Os que desconhecem, porém usariam corresponde a 29,41\% ( $\mathrm{n}=10$ ), os que desconhecem o uso de determinado produto natural para a melhoria do controle glicêmico e não usaria foi de $11,76 \%(\mathrm{n}=4)$. 
Uma parcela significativa de $29,41 \%$ ( $\mathrm{n}=10$ ) do estudo não soube opinar.

Neste item foi perguntada de maneira específica a adesão de produto natural para o tratamento de feridas e úlceras do pé diabético, embora seja semelhante às perguntas anteriores, este item é específico às feridas do pé diabético, enquanto a outra é referida a ferida genérica de múltiplas etiologias como as de causas infecciosas, traumáticas e lesões autoimunes. 20,59\% $(n=7)$ referiu que conhece e já fez uso ou usaria. 8,82\% $(n=3)$ referiu conhecer, porém não fez uso e nem pretende usá-los com finalidade terapêutica. Desconhece, entretanto, caso tivesse acesso faria uso correspondeu a uma taxa de 32,35\% (n=11) sendo a resposta majoritária. Desconhece e não usaria atingiu o valor de $8,82(n=3)$ e os que não souberam opinar representou $29,41 \%(\mathrm{n}=10)$.

A apresentação dos produtos terapêuticos normalmente varia de acordo com a característica anatômica do sítio de atuação e também das características farmacocinéticas e de manipulação da matéria-prima, isso a nível industrial. Neste item do questionário foi perguntado quanto à apresentação do produto natural na qual conheciam ou havia feito uso, foi majoritário o uso de infusões 20,59\% ( $n=7$ ) que pode estar na forma de chá, para uso interno ou banhos e aplicações tópicas. Em seguida, preparos de extratos embebidos em solução alcoólica registrou 17,65\% (n=6), enquanto extratos puros representaram 5,88\% $(\mathrm{n}=2)$. A apresentação in natura foi relatada por apenas um paciente, correspondendo a 2,94\%. As formas em pomadas, cremes ou sabonetes não foram citadas. 52,94\% $(\mathrm{n}=18)$ relataram desconhecer.

Foi citado entre o público desta pesquisa, diversos produtos naturais em apresentações distintas e com aplicações terapêuticas diferentes, algumas por via tópica e outras por uso oral com ação hipoglicemiante e/ou cicatrizante, principalmente as infusões/chás. Nas infusões/chás foi descrito a folha da amora (Morus sp.) que tem ação hipoglicemiante. Foram citados o uso do chá da folha de abacate (Persea americana) e o chá do chuchu (Sechium edule). Em uso tópico foi descrito pelos pacientes a folha da pata-de-vaca (Bauhinia sp.), contudo, nas apresentações em extratos associado a álcool foi descrito a raiz do sambacaitá (Hyptis sp.), do barbatimão (Stryphnodendron adstringens) e a folha de aroeira (Schinus terebinthifolius).

Quando perguntados sobre a eficácia dos produtos naturais em auxiliar o tratamento da síndrome do pé diabético, foi verificado que uma parte significativa admitiu uma visão positiva quanto à sua ação, de modo que $23,53 \%(\mathrm{n}=8)$ acreditam que seja muito provável efeitos benéficos com o uso de agentes naturais. Outra parcela de 23,53\% ( $\mathrm{n}=8$ ) acredita nos benefícios de forma provável. Pouco provável 8,82\% (n=3). Os que acreditam em improvável benefício representou 11,76\% (n=4). Não souberam opinar alcançou 32,35\% ( $\mathrm{n}=11)$. O último item questionou se os pacientes conheciam se o uso de determinados produtos naturais poderia oferecer ou causar algum tipo de problema ou agravar a situação dos pacientes que possuem úlceras decorrentes da síndrome do pé diabético e houve valores muito diferentes quanto às respostas; $94,12 \%$ dos pacientes $(\mathrm{n}=32)$ desconheciam qualquer risco e apenas 5,88\% $(n=2)$ citaram que poderia ocorrer reações alérgicas a nível da pele.

\section{Discussão}

Em relação a variável gênero, os números deste estudo não chegam a uma conclusão para um predomínio do gênero feminino como evidenciado por Marinho et al (2012) e Theme Filha et al. (2015), visto que a distribuição foi igual entre os sexos. Segundo Cortez et al. (2015), o paciente leva em torno de 10 anos ou mais para que ao serem diagnosticados com diabetes iniciem as complicações vasculares.

Neste estudo ficou evidente que quase $80 \%$ se consideravam sedentários, o que já conhece os agravos deste comportamento nas condições metabólicas e cardiovasculares, cujo associou em 77,14\% dos casos associados a hipertensão arterial, além de uma minoria, mas não menos significativa que já possuía cardiopatias crônicas e eram pacientes nefropatas (Malfatti \& Assunção, 2011). 
Em relação aos fatores de risco, estudos epidemiológicos evidenciaram que as doenças crônicas não transmissíveis como a associação da hipertensão arterial e o diabetes está comumente associada ao desenvolvimento de complicações crônicas como o pé diabético e acidente vascular encefálico (Souza et al., 2015).

Quanto ao nível de escolaridade este estudo apresentou divergências comparado ao estudo de Pitanga e Lessa (2005), que apontou como maioria analfabeta ou tinha ensino fundamental incompleto, enquanto neste estudo a maioria possui ensino médio completo, inferindo que mesmo nas populações com maior escolaridade a prevalência do diabetes e suas complicações aumenta, devido ao acesso a determinados alimentos e a menor prática de exercícios físicos e aos novos hábitos de vida. A elevada prevalência em populações com menor escolaridade pressupõe que as comunidades carentes ou de baixa renda já se incluem no processo de transição nutricional, geralmente acompanhada de uma trilogia de comorbidades (HAS, diabetes tipo II e sobrepeso/obesidade) que muito comumente, estão em conjunto em populações mais vulneráveis (Melo et al., 2020). Não foi encontrada na literatura fontes que explicassem os fatores hereditários de ordem paterna ou materna.

Medeiros et al. (2014) descreveram que os principais sintomas do diabetes são poliúria, xerostomia e polidipsia, além de perda de peso de forma abrupta nas fases mais avançadas, porém o diabetes, geralmente, é assintomático e pode apresentar sintomas que são desconsiderados e ignorados pelo indivíduo ou despercebidos pelas estratégias de atenção básica nos pacientes que são acompanhados. Nesse contexto, a suspeita clínica pode ocorrer a partir dos fatores de risco para a doença.

Quanto a utilização de produtos biológicos, de acordo com Singh et al. (2013), os principais constituintes químicos encontrados nos produtos naturais são os flavonoides, terpenos e os polifenois. Algumas ervas são ricas em inibidores da $\alpha$ glucosidase, o que consiste na redução da absorção dos carboidratos e consequente redução da glicemia (Surya et al., 2014).

Em relação às plantas identificadas encontrou-se estudos que demonstram propriedades bioativas. A Morus $s p$. foi citada por pacientes como sendo um produto biológico com potencial propriedades cicatrizantes e no controle glicêmico, o chá das folhas é bastante utilizado pela população local para o tratamento de diabetes, colesterol, problemas cardiovasculares, obesidade e gota. A planta foi trazida para a região do vale do São Francisco por imigrantes japoneses, adaptando-se bem às condições de clima e solo (Oliveira et al., 2013). Também foi relatada pelos pacientes a Persea americana, segundo estudos de Ezejiofor et al. (2013) e Feijó et al. (2012), o extrato aquoso de Persea americana apresentou redução dos níveis de glicose no sangue de ratos diabéticos, e no mesmo estudo Feijó verificou que a Bauhinia sp. contradiz quanto à redução dos níveis glicêmicos. Recentemente, uma revisão integrativa descreveu propriedades bioativas de Persea americana por possui teores elevados de compostos fenólicos, flavonoides e carotenoides, estes compostos estão associados à prevenção de doenças ocasionadas pela presença de radicais livres (Almeida et al., 2018). Outra planta de uso terapêutico que se destaca é o barbatimão (Stryphnodendron barbatiman Martius) suas cascas são espessas e apresentam efeito adstringente, geralmente preparado em solução alcoólica, que contém como princípio ativo e ação farmacológica o tanino (Coelho et al., 2010). A aroeira-vermelha, Schinus terebinthifolius, que também foi relatada, é uma espécie nativa do Brasil, sendo muito utilizada para combater diversas doenças, pois apresenta, propriedades anti-inflamatórias e cicatrizantes (Azevedo et al., 2015).

\section{Conclusão}

O estudo conclui que o perfil do paciente com diabetes e evolução para síndrome do pé diabético vem se alterando, evidenciando um aumento da prevalência em uma população com maior nível de escolaridade. Além disso, observou-se uma discreta incidência no sexo feminino e associação com outras doenças crônicas, sendo a principal, a hipertensão arterial sistêmica.

A grande parcela de pacientes acometidos compreendia classes sociais desfavorecidas, sendo importante conhecer o perfil epidemiológico desse público para a estruturação e criação de políticas públicas de saúde tal qual consiga chegar à 
realidade destes. Observou-se também que muitos pacientes fazem uso da sabedoria popular e são referenciados cientificamente produtos naturais com possíveis propriedades bioativas e farmacológicas que podem ser empregadas no tratamento alternativo destas patologias e prevenindo o aparecimento de complicações.

\section{Agradecimentos}

Ao Hospital Geral do Estado pela colaboração e livre acesso e a todos pacientes que dedicaram um pouco do seu tempo, à beira do leito, colaborando com o ensino e à pesquisa.

\section{Referências}

Almeida, C. A. N., Ued, F. V., Almeida, C. C. J. N., Almeida, A. C. F., Del Ciampo, L. A., Silva, L. F. O., et al. (2018). Perfil nutricional e benefícios do azeite de abacate (Persea americana): uma revisão integrativa. Braz. J. Food Technol., 21, e2017214.

Alvim, N. A. T., Ferreira, M. A., Cabral, I. E., \& Filho, A. J. A. (2006). O uso de plantas medicinais como recurso terapêutico: das influências da formação profissional às implicações éticas e legais de sua aplicabilidade como extensão da prática de cuidar realizada pela enfermeira. Rev Lat Am Enfermagem, 14(3), 316-23.

Azevedo, C. F., Quirino, Z. G. M., \& Bruno, R. L. A. (2015). Estudo farmacobotânico de partes aéreas vegetativas de aroeira-vermelha (Schinus terebinthifolius Raddi, Anacardiaceae). Rev. Bras. Plantas Med., 17(1), 26-35.

Caiafa, J. S., Castro, A. A., Fidelis, C., Santos, V. P., Silva, E. S., \& Júnior, C. J. S. (2011). Atenção integral ao portador de pé diabético. J. Vasc. Bras, 10(4): 132.

Coelho, J. M., Antoniolli, A. B., Nunes e Silva, D., Carvalho, T. M. M. B., Pontes, E. R. J. C., \& Odashiro, A. N. (2010). O efeito da sulfadiazina de prata, extrato de ipê-roxo e extrato de barbatimão na cicatrização de feridas cutâneas em ratos. Rev. Col. Bras. Cir., 37(1), 45-51.

Cortez, D. N., Reis, I. A., Souza, D. A. S., Macedo, M. M. L., \& Torres, H. C. Complicações e o tempo de diagnóstico do diabetes mellitus na atenção primária. Acta Paul. Enferm., 28(3), 250-255.

Crawford, K. (2017). Review of 2017 Diabetes Standards of Care. Nursing Clinics Of North America, 52(4), 621-63.

Ezejiofor, A. N., Okorie, A., \& Orisakwe, O. E. (2013). Hypoglycaemic and tissue-protective effects of the aqueous extract of Persea americana seeds on alloxan-induced albino rats. The Malaysian journal of medical sciences: MJMS, 20(5), 31-39.

Feijó, A. M., Bueno, M. E. N., Ceolin, T., Linck, C. L., Schwartz, E., Lange, C., et al. (2012). Plantas medicinais utilizadas por idosos com diagnóstico de Diabetes mellitus no tratamento dos sintomas da doença. Rev. Bras. Plantas Med., 14(1), 50-56.

Franco, R. R., Carvalho, D. S., Rosa de Moura, F. B., Justino, A. B., Silva, H. C. G., Peixoto, L. G., et al. (2018). Antioxidant and anti-glycation capacities of some medicinal plants and their potential inhibitory against digestive enzymes related to type 2 diabetes mellitus. Journal of Ethnopharmacology, 215, 140146.

Malfatti, C. R. M., \& Assunção, A. N. (2011). Hipertensão arterial e diabetes na Estratégia de Saúde da Família: uma análise da frequência de acompanhamento pelas equipes de Saúde da Família. Ciênc. Saúde Coletiva, 16 (supl. 1), 1383-1388.

Marinho, N. B. P., Vasconcelos, H. C. A., Alencar, A. M. P. G., Almeida, P. C., \& Damasceno, M. M. C. (2012). Diabetes mellitus: fatores associados entre usuários da estratégia saúde da família. Acta Paul. Enfer., 25(4), 595-600.

Medeiros, P. M., Araújo, L. M. N., Brito, F. A. P., Salvador, P. T. C. O., Tourinho, F. S. V., Santos, V. E. P. (2014). Processo de cuidar do portador de diabetes mellitus: revisão integrativa da literatura. Comun. Ciênc. Saúde, 24(3), 251-258.

Melo, S. P. S. C., Cesse, E. A. P., Lira, P. I. C., Ferreira, L. C. C. N., Rissin, A., \& Filho, M. B. (2013). Sobrepeso, obesidade e fatores associados aos adultos em uma área urbana carente do Nordeste Brasileiro. Rev. Bras. Epidemiol., 23, e200036.

Oliveira, A. C. B., Oliveira, A. P., Guimarães, A. L., Oliveira, R. A., Silva, F. S., Reis, S. A. G. B., et al. (2013). Avaliação toxicológica pré-clínica do chá das folhas de Morus nigra L. (Moraceae). Rev. Bras. Plantas Med., 15(2), 244-249.

Pereira, A. S., Shitsuka, D. M., Parreira, F. J., \& Shitsuka, R. (2018). Metodologia da pesquisa científica. UFSM, 119p.

Pitanga, F. G. J., \& Lessa, I. (2005). Prevalência e fatores associados ao sedentarismo no lazer em adultos. Cad. Saúde Pública, $21(3)$, 870-877.

Sagbo, I. J., Afolayan, A. J., \& Bradley, G. (2017). Antioxidant, antibacterial and phytochemical properties of two medicinal plants against the wound infecting bacteria. Asian Pac. J. Trop. Biomed, 7(9): 817-825.

Sartorelli, D. S., \& Franco, L. J. (2003). Tendências do diabetes mellitus no Brasil: o papel da transição nutricional. Cad. Saúde Pública, 19(Sup. 1), 29-36.

Singh, R., Kaur, N., Kishore, L., \& Gupta, G. K. (2013). Management of diabetic complications: A Chemical constituents based approach. J Ethnopharmacol, 150(1), 51-70. 
Research, Society and Development, v. 10, n. 3, e2110313113, 2021

(CC BY 4.0) | ISSN 2525-3409 | DOI: http://dx.doi.org/10.33448/rsd-v10i3.13113

Souza, N. P. G., Oliveira, G. Y. M., Girão, A. L. A., Souza, L. M., Maniva, S. J. C. F., Freitas, C. H. A. (2015). Adoecimento por hipertensão arterial e Diabetes Mellitus: concepções de um grupo de pacientes hospitalizados. Rev Enferm. UERJ, 23(1), 52-57.

Surya, S., Salam, A. D., Tomy, D. V., Carla, B., Kumar, R. A., \& Sunil. C. (2014). Diabetes mellitus and medicinal plants - a review. Asian Pacific Tropical Medicine Press, 4(5), 337-347.

Theme Filha, M. M., Junior, P. R. B. S., Damacena, G. N., \& Szwarcwald, C. L. Prevalência de doenças crônicas não transmissíveis e associação com autoavaliação de saúde: Pesquisa Nacional de Saúde. 2013. Rev. Bras. Epidemiol., 18 (supl.2), 83-96. 